\title{
DVCS-dissociation of the deuteron and the EMC effect
}

\author{
Silas R. Beane ${ }^{\mathrm{a}, \mathrm{b}, *}$, Martin J. Savage $\mathrm{c}^{\mathrm{c}, \mathrm{d}}$ \\ a Department of Physics, University of New Hampshire, Durham, NH 03824-3568, USA \\ b Jefferson Laboratory, 12000 Jefferson Avenue, Newport News, VA 23606, USA \\ c Department of Physics, University of Washington, Seattle, WA 98195-1560, USA \\ d Center for Theoretical Physics, MIT, Cambridge, MA 02139, USA
}

Received 7 January 2005; received in revised form 8 June 2005; accepted 21 July 2005

Available online 15 August 2005

\begin{abstract}
The break-up of the deuteron during deeply-virtual Compton scattering, $\gamma^{*} d \rightarrow \gamma^{(*)} n p$, is explored. In the effective field theory describing nucleon dynamics at momenta below the pion mass, the EMC effect results from four-nucleon interactions with the twist- 2 operators, appropriate for describing forward, and near-forward, matrix elements in the two-nucleon system. We point out that the break-up of the deuteron to low-energy final states during deeply-virtual Compton scattering is a process with which to explore strong-interaction physics closely related to that responsible for the EMC effect. The single-nucleon contribution to the break-up depends on the moments of the spin-dependent structure functions and contributions from local four-nucleon operators. Experimental deviations from the single-nucleon prediction would provide a probe of strong interactions complimentary to the EMC effect.

() 2005 Elsevier B.V. All rights reserved.
\end{abstract}

A naive glance at QCD would suggest that the nucleus is a non-perturbative collection of quarks and gluons with no discernible structure. However, this is not the case, and a remarkable aspect of nuclear physics is that nuclei may be viewed as a collection of relatively weakly-interacting nucleons. The small binding energy of the deuteron implies that

\footnotetext{
* Corresponding author.

E-mail address: silas@physics.unh.edu (S.R. Beane). 
it very-nearly resembles a free proton and neutron; its magnetic moment is very close to the sum of proton and neutron magnetic moments, and its structure functions are very close to the sum of the nucleon structure functions. Deviations from the single-nucleon structure functions, known as the EMC effect, ${ }^{1}$ were experimentally observed [1] in nuclei some twenty years ago and caused a great deal of excitement, e.g., [2-5]. However, as the nucleons do interact, albeit weakly, one expects matrix elements of all electro-weak probes to be modified from their single-nucleon values, and the EMC effect should be considered to be nothing more than another demonstration of this. Significant efforts have gone into quantitatively explaining the EMC effect in explicit models of nuclei, e.g., Refs. [6,7]. One would like to uncover the dominant strong-interaction mechanism that allows for a quantitative explanation of the effect, but given that one cannot accurately predict the deuteron quadrupole moment from modern potential models ${ }^{2}$ to better than $\sim 5 \%$, an approximate explanation would suffice at present.

Within the framework of the pionless effective field theory, EFT( $\not t)$, it is easy to understand and describe the EMC effect. By constructing all the operators required to reproduce the matrix elements of the twist- 2 operators in multi-nucleon systems, one sees that operators involving more than one nucleon are not forbidden by the symmetries of the strong interaction, and therefore must be present. While observation of the EMC effect twenty years ago may have been surprising to some, in fact, its absence would have been far more surprising. In this work we write down the most general set of operators describing the interaction between two nucleons in an S-wave that reproduce the matrix elements of the twist-2 operators. As the origin of the EMC effect is strong-interaction physics at distance scales of order the Compton wavelength of the pion or smaller, there will be an analogous deviation from the single-nucleon prediction for transitions between two nucleons in the ${ }^{3} S_{1}$ channel and the ${ }^{1} S_{0}$ channel. Therefore, we suggest that an experimental determination of the break-up of the deuteron during deeply-virtual compton scattering (DVCS), with very low-energy final-state nucleons, provides a probe of a purely-nuclear effect which is complimentary to the EMC effect.

In any given hadron, the moments of the parton distribution functions are given by

$$
\begin{aligned}
\left\langle x^{n-1}\right\rangle_{q_{V}} & =\int_{0}^{1} d x x^{n-1}\left(q(x)+(-1)^{n} \bar{q}(x)\right), \\
\left\langle x^{n-1}\right\rangle_{q_{A}} & =\int_{0}^{1} d x x^{n-1}\left(\Delta q(x)+(-1)^{n-1} \Delta \bar{q}(x)\right),
\end{aligned}
$$

\footnotetext{
1 The expression "EMC effect" usually refers to deviations from single-nucleon structure in nuclei with $A \gg 2$. Here we apply the expression to the deuteron and therefore, in the present context, the EMC effect is relevant to extractions of the neutron structure functions.

2 The quadrupole moment of the deuteron is successfully described in effective field theory by the inclusion of a local operator at next-to-leading order in the expansion [8]. The discrepancy between potential model calculations and the experimental value is due to the omission of physics at distance scales smaller than the Compton wavelength of the pion, which may or may not be describable by a meson-exchange mechanism.
} 
where $q(\bar{q})$ is the quark (antiquark) spin-averaged distribution and $\Delta q(\Delta \bar{q})$ is the helicity distribution (we do not consider the transversity distribution). From QCD, these moments have the clear interpretation as the forward matrix elements of local operators,

$$
\begin{aligned}
& \theta_{V, \mu_{1} \ldots \mu_{n}}^{(n), 0}=(i)^{n-1} \bar{q} \gamma_{\left\{\mu_{1}\right.} \overleftrightarrow{D}_{\mu_{2}} \cdots \overleftrightarrow{D}_{\left.\mu_{n}\right\}} q, \\
& \theta_{V, \mu_{1} \ldots \mu_{n}}^{(n), b}=(i)^{n-1} \bar{q} \gamma_{\left\{\mu_{1}\right.} \overleftrightarrow{D}_{\mu_{2}} \cdots \overleftrightarrow{D}_{\left.\mu_{n}\right\}} \tau^{b} q, \\
& \theta_{A, \mu_{1} \ldots \mu_{n}}^{(n), 0}=(i)^{n-1} \bar{q} \gamma_{\left\{\mu_{1}\right.} \overleftrightarrow{D}_{\mu_{2}} \cdots \overleftrightarrow{D}_{\left.\mu_{n}\right\}} \gamma_{5} q, \\
& \theta_{A, \mu_{1} \ldots \mu_{n}}^{(n), b}=(i)^{n-1} \bar{q} \gamma_{\left\{\mu_{1}\right.} \overleftrightarrow{D}_{\mu_{2}} \cdots \overleftrightarrow{D}_{\left.\mu_{n}\right\}} \gamma_{5} \tau^{b} q,
\end{aligned}
$$

where the indices enclosed by $\{\cdots\}$ are symmetrized and their traces are removed. An analysis of matrix elements of these operators in the single-nucleon sector has been carried out at the one-loop level in the chiral expansion [9-13]. Those works have been extended from QCD to quenched QCD [14] and partially-quenched QCD [15,16] for applications to lattice QCD simulations. In addition to forward matrix elements of these operators, offforward matrix elements have been considered [17], and the issue of soft-pion production during DVCS has been explored [18-20]. For very low-energy processes, those involving momentum and energy transfers much less than the pion mass, one can work in EFT $(\not t)$. In this energy regime, the matrix elements of the operators in Eq. (2) between single-nucleon states are reproduced by

$$
\begin{aligned}
& \theta_{V, \mu_{1} \ldots \mu_{n}}^{(n), 0} \rightarrow M^{n-1}\left\langle x^{n-1}\right\rangle_{q_{V}^{(0)}} v_{\left\{\mu_{1}\right.} v_{\mu_{2}} \cdots v_{\left.\mu_{n}\right\}} \bar{N} N+\cdots, \\
& \theta_{V, \mu_{1} \ldots \mu_{n}}^{(n), b} \rightarrow M^{n-1}\left\langle x^{n-1}\right\rangle_{q_{V}^{(1)}} v_{\left\{\mu_{1}\right.} v_{\mu_{2}} \cdots v_{\left.\mu_{n}\right\}} \bar{N} \tau^{b} N+\cdots, \\
& \theta_{A, \mu_{1} \ldots \mu_{n}}^{(n), 0} \rightarrow M^{n-1}\left\langle x^{n-1}\right\rangle_{q_{A}^{(0)}} v_{\left\{\mu_{1}\right.} v_{\mu_{2}} \cdots v_{\mu_{n-1}} \bar{N} S_{\left.\mu_{n}\right\}} N+\cdots, \\
& \theta_{A, \mu_{1} \ldots \mu_{n}}^{(n), b} \rightarrow M^{n-1}\left\langle x^{n-1}\right\rangle_{q_{A}^{(1)}} v_{\left\{\mu_{1}\right.} v_{\mu_{2}} \cdots v_{\mu_{n-1}} \bar{N} S_{\left.\mu_{n}\right\}} \tau^{b} N+\cdots,
\end{aligned}
$$

where $\left\langle x^{p}\right\rangle_{q_{V}^{(0)}}$ and $\left\langle x^{p}\right\rangle_{q_{A}^{(0)}}$ are the $p$ th moments of the isoscalar vector and axial-vector PDF's in the nucleon, respectively, and $\left\langle x^{p}\right\rangle_{q_{V}^{(1)}}$ and $\left\langle x^{p}\right\rangle_{q_{A}^{(1)}}$ are the $p$ th moments of the isovector PDF's in the nucleon, respectively. $M$ is the nucleon mass. For $n=1$ baryon-number conservation gives $\langle 1\rangle_{q_{V}^{(0)}}=3$; isospin-conservation gives $\langle 1\rangle_{q_{V}^{(1)}}=1$; and matching to the axial-vector currents gives $\langle 1\rangle_{q_{A}^{(0)}}=g_{0}$, the singlet axial charge, ${ }^{3}$ and $\langle 1\rangle_{q_{A}^{(1)}}=g_{A}$, the isovector axial charge. The nucleon four-velocity is $v$, and in the nucleon rest frame becomes $v=(1,0,0,0)$. The ellipses denote terms that are higher order in the derivative expansion, suppressed by powers of $p / m_{\pi}$.

When considering the parton distributions of the deuteron, the simplest nucleus, insertions of the operators of Eq. (3) defines the "one-body operator truncation" or OBOT.

\footnotetext{
3 The " $q$ " subscripts on the operator coefficients in Eq. (3) and Eq. (4) indicate that we are considering matrix elements of the quark operators only, and not the purely-gluonic operators-including the anomaly-which mix with the quark operators under renormalization. The singlet axial charge defined here, $g_{0}$, is therefore scale dependent.
} 
Given the operator structure, it is clear that one simply recovers the naive sum of contributions from the proton and neutron. However, it is also clear that, in the effective field theory expansion, there are contributions from higher-dimensional operators involving more nucleons. The leading-order interactions involving two-nucleons are

$$
\begin{aligned}
\theta_{V, \mu_{1} \ldots \mu_{n}}^{(n), 0} \rightarrow & M^{n-1}\left[\alpha_{q}^{(n)} v_{\left\{\mu_{1}\right.} v_{\mu_{2}} \cdots v_{\left.\mu_{n}\right\}}\left(N^{T} P_{1}^{a} N\right)^{\dagger} N^{T} P_{1}^{a} N\right. \\
& +\beta_{0 ; q}^{(n)} v_{\left\{\mu_{1}\right.} v_{\mu_{2}} \cdots v_{\left.\mu_{n}\right\}}\left(N^{T} P_{3}^{\alpha} N\right)^{\dagger} N^{T} P_{3}^{\alpha} N \\
& \left.+\beta_{2 ; q}^{(n)} v_{\left\{\mu_{3}\right.} v_{\mu_{4}} \cdots v_{\mu_{n}}\left(N^{T} P_{3}^{\mu_{1}} N\right)^{\dagger} N^{T} P_{3}^{\left.\mu_{2}\right\}} N\right], \\
\theta_{V, \mu_{1} \ldots \mu_{n}}^{(n), a} \rightarrow & M^{n-1} \gamma_{q}^{(n)} i \varepsilon^{a b c} v_{\left\{\mu_{1}\right.} v_{\mu_{2}} \cdots v_{\left.\mu_{n}\right\}}\left(N^{T} P_{1}^{b} N\right)^{\dagger} N^{T} P_{1}^{c} N, \\
\theta_{A, \mu_{1} \ldots \mu_{n}}^{(n), 0} \rightarrow & M^{n-1} \rho_{q}^{(n)} v_{\left\{\mu_{1}\right.} v_{\mu_{2}} \cdots v_{\mu_{n-1}} i \varepsilon^{\left.\mu_{n}\right\} \alpha \beta \gamma} v_{\alpha}\left(N^{T} P_{3}^{\beta} N\right)^{\dagger} N^{T} P_{3}^{\gamma} N, \\
\theta_{A, \mu_{1} \ldots \mu_{n}}^{(n), a} \rightarrow & M^{n-1} \sigma_{q}^{(n)} v_{\left\{\mu_{1}\right.} v_{\mu_{2}} \cdots v_{\mu_{n-1}}\left(N^{T} P_{1}^{a} N\right)^{\dagger} N^{T} P_{3}^{\left.\mu_{n}\right\}} N+\text { h.c. },
\end{aligned}
$$

where with $n=1$ we have $\beta_{2 ; q}^{(1)}=0$ (due to available Lorentz indices) and baryon-number conservation gives $\alpha_{q}^{(1)}=\beta_{0 ; q}^{(1)}=0$; isospin-conservation gives $\gamma_{q}^{(1)}=0$; and matching to the axial-vector current gives $\rho_{q}^{(1)}=-2 L_{2, A}$ and $\sigma_{q}^{(1)}=L_{1, A}[21] .{ }^{4}$ The objects $P_{1}^{a}$ and $P_{3}^{\mu}[22-24]$ are the spin-isospin projectors for the ${ }^{1} S_{0}$ and ${ }^{3} S_{1}$ channels, respectively,

$$
P_{1}^{a}=\frac{1}{\sqrt{2}} \tau_{2} \tau^{a} \otimes S_{2}, \quad P_{3}^{\mu}=\sqrt{2} \tau_{2} \otimes S_{2} S^{\mu},
$$

where $S^{\mu}$ is the usual spin operator which acts on the nucleon fields. The contributions of such operators to the parton distributions of the deuteron can be computed straightforwardly in $\mathrm{EFT}(\not t)[8,25] .{ }^{5}$ However, it is convenient to switch notation to that of EFT( $\left.\not t\right)$ constructed in terms of di-baryon fields [26,27], dEFT $(\not t)$, in which the leading effects of the finite effective range are resummed.

In this work we will discuss relevant aspects of the di-baryon formalism, dEFT( $\not t$ ) [26$28]$. In terms of nucleon and di-baryon degrees of freedom, the leading-order low-energy strong interactions for $|\mathbf{p}| \ll m_{\pi} / 2$ are described by a Lagrange density of the form

$$
\begin{aligned}
\mathcal{L}= & N^{\dagger}\left[i \partial_{0}+\frac{\nabla^{2}}{2 M}\right] N+t_{\mu}^{\dagger}\left[i \partial_{0}+\frac{\nabla^{2}}{4 M}-\Delta_{3}\right] t^{\mu} \\
& -s_{a}^{\dagger}\left[i \partial_{0}+\frac{\nabla^{2}}{4 M}-\Delta_{1}\right] s^{a}+y_{3}\left[t_{\mu}^{\dagger} N^{T} P_{3}^{\mu} N+\text { h.c. }\right] \\
& -y_{1}\left[s_{a}^{\dagger} N^{T} P_{1}^{a} N+\text { h.c. }\right],
\end{aligned}
$$

\footnotetext{
4 The cross sections for the weak disintegration of the deuteron and other two-nucleon weak processes depend on the parameter $L_{1, A}$. The axial operator with coefficient $L_{2, A}$ contributes-together with the sum of the neutron and proton contributions - to the strangeness content of the deuteron.

5 The effective field theory can be constructed in terms of operators that do not vanish on the particle massshells. This is true for any consistent theory, and operators that only contribute off mass-shell can always be eliminated.
} 
where $N$ is the nucleon annihilation operator, we have used the four-vector $t_{\mu}=\left(0, t_{j}\right)$ with $t^{j}$ the ${ }^{3} S_{1}$ di-baryon annihilation operator with spin-index $j$, and $s^{a}$ is the ${ }^{1} S_{0}$ dibaryon annihilation operator with isospin-index $a$. In the Lagrange density in Eq. (6), a factor of the nucleon mass $M(2 M)$ has been absorbed into the definition of the nucleon fields (di-baryon fields). The $\mathrm{S}$-wave interactions are enhanced by a factor of the expansion parameter, $1 / p$, and are treated non-perturbatively. However the interactions that induce mixing with higher partial waves, e.g., ${ }^{3} S_{1}-{ }^{3} D_{1}$ mixing, are suppressed by at least $p^{2}$ and so only $\mathrm{S}$-wave to $\mathrm{S}$-wave interactions are required to the order we are working. Note however that the effects of the D-state from momenta of order or greater than the pion mass are present in the coefficients of leading-order operators. To recover the scattering amplitudes in both S-wave channels, the constants that appear in Eq. (6) are found to be [26]

$$
\begin{aligned}
& y_{3}^{2}=\frac{8 \pi}{M^{2} r_{3}}, \quad y_{1}^{2}=\frac{8 \pi}{M^{2} r_{1}}, \\
& \Delta_{3}=\frac{2}{M r_{3}}\left(\frac{1}{a_{3}}-\mu\right), \quad \Delta_{1}=\frac{2}{M r_{1}}\left(\frac{1}{a_{1}}-\mu\right),
\end{aligned}
$$

where $\mu$ is the renormalization scale, $a_{3}$ and $r_{3}$ are the scattering length and effective range in the ${ }^{3} S_{1}$ channel, and $a_{1}$ and $r_{1}$ are the scattering length and effective range in the ${ }^{1} S_{0}$ channel. Insertions of the twist-2 operators give rise to operators of the form

$$
\begin{aligned}
\theta_{V, \mu_{1} \ldots \mu_{n}}^{(n), 0} \rightarrow & M^{n-1}\left[\tilde{\alpha}_{q}^{(n)} v_{\left\{\mu_{1}\right.} v_{\mu_{2}} \cdots v_{\left.\mu_{n}\right\}} s_{a}^{\dagger} s^{a}+\tilde{\beta}_{0 ; q}^{(n)} v_{\left\{\mu_{1}\right.} v_{\mu_{2}} \cdots v_{\left.\mu_{n}\right\}} t_{\alpha}^{\dagger} t^{\alpha}\right. \\
& \left.+\tilde{\beta}_{2 ; q}^{(n)} v_{\left\{\mu_{3}\right.} v_{\mu_{4}} \cdots v_{\mu_{n}} t_{\mu_{1}}^{\dagger} t^{\left.\mu_{2}\right\}}\right] \\
\theta_{V, \mu_{1} \ldots \mu_{n}}^{(n), 3} \rightarrow & M^{n-1} \tilde{\gamma}_{q}^{(n)} i \varepsilon^{3 b c} v_{\left\{\mu_{1}\right.} v_{\mu_{2}} \cdots v_{\left.\mu_{n}\right\}} s_{b}^{\dagger} s_{c} \\
\theta_{A, \mu_{1} \ldots \mu_{n}}^{(n), 0} \rightarrow & M^{n-1} \tilde{\rho}_{q}^{(n)} v_{\left\{\mu_{1}\right.} v_{\mu_{2}} \cdots v_{\mu_{n-1}} i \varepsilon^{\left.\mu_{n}\right\} \alpha \beta \gamma} v_{\alpha} t_{\beta}^{\dagger} t_{\gamma}, \\
\theta_{A, \mu_{1} \ldots \mu_{n}}^{(n), 3} \rightarrow & M^{n-1} \tilde{\sigma}_{q}^{(n)} v_{\left\{\mu_{1}\right.} v_{\mu_{2}} \cdots v_{\mu_{n-1}}\left[s_{3}^{\dagger} t^{\left.\mu_{n}\right\}}+\text { h.c. }\right] .
\end{aligned}
$$

With $n=1$ we have $\tilde{\beta}_{2 ; q}^{(1)}=0$ and baryon-number conservation gives $\tilde{\alpha}_{q}^{(1)}=-\tilde{\beta}_{0 ; q}^{(1)}=6$; isospin-conservation gives $\tilde{\gamma}_{q}^{(1)}=2$; and matching to the axial-vector current gives $\tilde{\rho}_{q}^{(1)}=$ $-2 \ell_{2, A} / 2 M \sqrt{r_{1} r_{3}}$ and $\tilde{\sigma}_{q}^{(1)}=\ell_{1, A} / 2 M \sqrt{r_{1} r_{3}}$ [29]. (Recent work has shown that it is possible to compute the coefficients of the operators in Eq. (8) in lattice QCD by making use of background-field methods [29,30].)

It is now straightforward to determine the matrix elements of the relevant operators between deuteron states. Keeping only the $\Delta L=0$ contribution from the diagrams shown in Fig. 1, the matrix elements of the isoscalar-vector quark operators at momentum transfer $\mathbf{q} \ll m_{\pi}$ are

$$
\left\langle d\left|\theta_{V, \mu_{1} \ldots \mu_{n}}^{(n), 0}\right| d\right\rangle=M^{n-1} v_{\left\{\mu_{1}\right.} v_{\mu_{2}} \cdots v_{\left.\mu_{n}\right\}}\left[2\left\langle x^{n-1}\right\rangle_{q_{V}^{(0)}} F_{C}(|\mathbf{q}|)-\tilde{\eta}_{0 ; q}^{(n)} \frac{\gamma r_{3}}{1-\gamma r_{3}}\right],
$$

where the expression is given in terms of the electric charge form factor

$$
F_{C}(|\mathbf{q}|)=\frac{\gamma r_{3}}{1-\gamma r_{3}}\left[\frac{4}{|\mathbf{q}| r_{3}} \tan ^{-1}\left(\frac{|\mathbf{q}|}{4 \gamma}\right)-1\right] .
$$



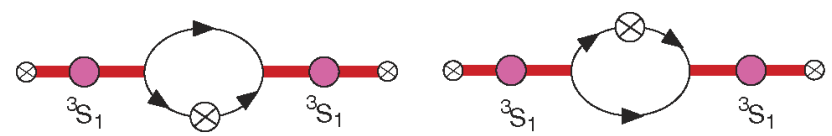

(a)

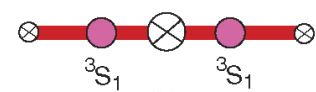

(b)

Fig. 1. Diagrams in $\mathrm{dEFT}(\not t)$ that provide the leading-order contribution to the matrix elements of the twist-2 operators in the deuteron. The thick solid lines with solid circles at their midpoint correspond to fully-dressed di-baryons, while the thin lines correspond to nucleons. The crossed circles correspond to insertions of the twist-2 operators.

The single-nucleon contribution has been made explicit by writing

$$
-\tilde{\beta}_{0 ; q}^{(n)}=2\left\langle x^{n-1}\right\rangle_{q_{V}^{(0)}}+\tilde{\eta}_{0 ; q}^{(n)} .
$$

Here we see explicitly that the contribution from the nucleon structure functions (first term in the square brackets of Eq. (9)) is accompanied by a nuclear contribution (second term in the square brackets of Eq. (9)), making clear the origin of the EMC effect. Notice that for the baryon-number current $(n=1), \tilde{\eta}_{0 ; q}^{(1)}=0$ and the matrix element of Eq. (9) is entirely given by the leading-order electric charge form factor of the deuteron; as emphasized in Ref. [28], it is a peculiar feature of $\operatorname{dEFT}(\not t)$ (unlike $\operatorname{EFT}(\not t)$ ) that higher-dimensional local di-baryon operators contain contributions that should be interpreted as leading-order in the effective field theory expansion. Several points are worth emphasizing as they bear on conventional explanations of the EMC effect: (1) relativistic corrections appear at the next order in the effective field theory expansion and can be systematically included. These corrections have been computed for various deuteron properties in EFT( $\not t)$ and found to be small [8]; for static processes these corrections scale as $\gamma^{2} / M^{2}$. Notice that this expansion parameter is suppressed relative to the expansion parameter $\gamma / m_{\pi}$ characteristic of the size of the purely nuclear effects in $\operatorname{EFT}(\not t)$ and $\mathrm{dEFT}(\not t)$. (2) "Fermi motion" (or more appropriately, "zero-point energy") of the deuteron, is included in EFT( $\not t)$ and $\operatorname{dEFT}(\not t)$; terms of the form $\vec{p}^{2} / 2 m$ where $\vec{p}$ is a nucleon momentum are summed to all orders through the non-relativistic nucleon propagators from Eq. (6). (3) As pointed out above, in $\mathrm{EFT}(\not t)$ and $\mathrm{dEFT}(\not t)$ D-state admixtures enter at higher order in the effective field theory expansion [8].

There is also a "tensor EMC effect". This is induced by the operator in Eq. (8) with coefficient $\tilde{\beta}_{2 ; q}^{(n)}$, and corresponds to transferring $\Delta L=2$ to the hadronic system. This does not contribute to DIS, as it is not a forward matrix element, but will contribute to DVCS. If the deuteron polarization in the final state could be measured after DVCS on a polarized deuteron target, then in principle this object could be measured. Experimentally, such a measurement will not be easy. 

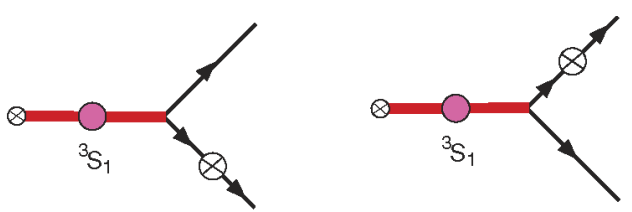

(a)
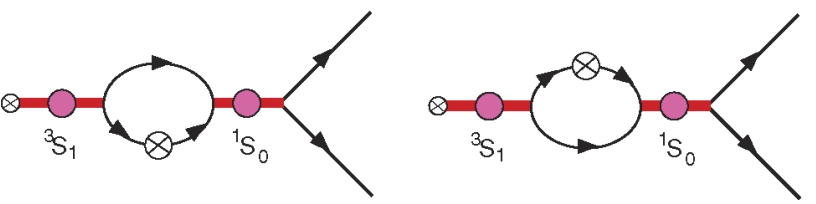

(b)

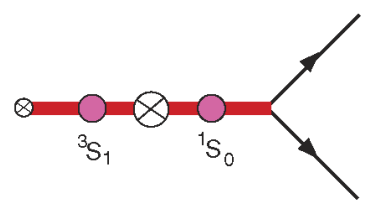

(c)

Fig. 2. Diagrams in dEFT( $\not$ ) that provide the leading-order contribution to the matrix elements for the break-up of the deuteron during DVCS. The thick solid lines with solid circles at their midpoint correspond to fully-dressed di-baryons, while the thin lines correspond to nucleons. The crossed circles correspond to insertions of the twist-2 operators.

A different situation in which the effects of the interactions between nucleons during DVCS will lead to modifications to the OBOT is the break-up of the deuteron. ${ }^{6}$ Such deviations are, in some sense, intimately related to the EMC effect as they result from different four-nucleon operators connecting S-wave states. They are analogous to the local four-nucleon operators which cause deviations from the OBOT for low-energy $n p \rightarrow d \gamma$ (which result from interactions between nucleons that are not related by gauge-invariance to the nucleon-nucleon scattering amplitude). It may or may not be the case that pionexchange currents can reproduce much of this contribution.

It is straightforward to show that for very-low energy and momentum transfers to the hadronic system, the amplitude for DVCS-dissociation of the deuteron can be written as

$$
\mathcal{A}=X M^{n-1} v_{\left\{\mu_{1}\right.} v_{\mu_{2}} \cdots v_{\mu_{n-1}} \varepsilon_{\left.\mu_{n}\right\}}^{(d)} U_{n}^{T} \sigma_{2} U_{p},
$$

for the neutron and proton in a ${ }^{1} S_{0}$ final-state, where $\varepsilon_{\mu}^{(d)}$ is the deuteron polarization vector, and $U_{n, p}$ are the spinors associated with the neutron and proton respectively. For the sake of simplicity, we give an expression for the coefficient $X$ in Eq. (12), resulting from the diagrams in Fig. 2, for kinematics where the energy-transfer and momentum-transfer to the hadronic system are equal, i.e., light-like. We find

\footnotetext{
6 For a recent discussion of the process $e+d \rightarrow e+n+p$ in the $1.5 \leqslant Q^{2}<4 \mathrm{GeV}^{2}$ range, see Ref. [31]. Deviations from the OBOT in DVCS on nuclei has been considered recently in Refs. [32,33].
} 


$$
\begin{aligned}
X= & 2 i \sqrt{\frac{\pi}{\gamma^{3}}} \frac{1}{\sqrt{1-\gamma r_{3}}} \frac{1}{\left[-\frac{1}{a_{1}}+\frac{1}{2} r_{1}|\mathbf{p}|^{2}-i|\mathbf{p}|\right]} \\
& \times\left[\left\langle\left. x^{n-1}\right|_{q_{A}^{(1)}} \frac{\gamma^{2}}{|\mathbf{p}|^{2}+\gamma^{2}}\left(\gamma-\frac{1}{a_{1}}+\frac{1}{2} r_{1}|\mathbf{p}|^{2}\right)+\frac{1}{2} \gamma^{2} \sqrt{r_{1} r_{3}} \tilde{\sigma}_{q}^{(n)}\right],\right.
\end{aligned}
$$

where $\mathbf{p}$ is the momentum of one of the nucleons in the final state. ${ }^{7}$ While the spindependent structure functions determine the single-nucleon contributions to the deuteron break-up (first term in the square brackets of Eq. (13) proportional to $\left\langle x^{n-1}\right\rangle_{q_{A}^{(1)}}$ ), there is an additional contribution coming from short-distance strong interactions (second term in the square brackets of Eq. (13) proportional to $\tilde{\sigma}_{q}^{(n)}$ ). This amplitude is similar in form to that of $n p \rightarrow d \gamma$ [34,35] for obvious reasons. ${ }^{8}$

We emphasize that the validity of $\operatorname{dEFT}(\not t)$ requires that the energy imparted to the hadronic system be significantly less than $m_{\pi}$. For higher energies, pions must be included explicitly in the description. Even with the inclusion of pions as dynamical fields, local four-nucleon operators will continue to contribute to nuclear matrix elements of the twist2 operators. In the pionful theory these operators describe contributions from physics at scales of the order of and smaller than the chiral symmetry breaking scale. It is important to point out that the same two-body operators that are inserted in the deuteron will contribute to DVCS on the triton and ${ }^{3} \mathrm{He}$, with three-body operators expected to be suppressed by their naive engineering dimensions $[36,37] .^{9}$

In this work we have discussed the EMC effect in terms of the matrix elements of local operators. We have made no attempt to determine the underlying mechanism; rather we have demonstrated how to describe the effect in a model-independent manner. As for the case of the single nucleon, only positive moments of the parton distributions can be related to the forward matrix elements of local operators, and so it is natural to consider only the positive moments of the parton distributions of nuclei, including the deuteron. By contrast, the small- $x$ behavior is not related to forward matrix elements of local operators, and arises from long-distance strong-interaction contributions to the electromagnetic-nucleus interactions. So while the positive moments can be considered properties of nucleons and nuclei, the small- $x$ behavior is a property of the interaction matrix element, and in general, is different for each probe. For forward processes and those involving only small momentum transfers we have used EFT( $\not t)$ and $\mathrm{dEFT}(\not t)$ to compute the EMC effect and the DVCS-dissociation of the deuteron in terms of single-nucleon contributions and fournucleon interactions due to short-distance physics. We consider this paper to be the first step toward a detailed calculation of the dissociation process with realistic kinematics. We estimate that a measurement of the break-up cross sections will show deviations from the single-particle estimate at the level of $5 \% \rightarrow 10 \%$, in analogy with the process $n p \rightarrow d \gamma$,

\footnotetext{
7 Notice that while this form-factor sums all orders in $|\mathbf{p}| / \gamma$, it is perturbative in the expansion parameter $|\mathbf{p}| / m_{\pi}$.

8 Both terms in the square brackets of Eq. (13) naively appear to be of the same order in the expansion, however one power of the expansion parameter, $p$, has been hidden in $\tilde{\sigma}_{q}^{(n)}$ for ease of notation.

9 This expectation for three-body operators is based on the fact that the two-body operators power count like a perturbative insertion of a local operator in the strong sector.
} 
and in keeping with the EMC effect. Measurement of this break-up cross section at Jefferson Laboratory or elsewhere, and a determination of the deviation from the single-nucleon effect, would provide a fascinating probe of the strong interactions.

\section{Acknowledgements}

We are grateful to Jerry Miller for comments and discussions which have substantially increased the quality of the manuscript. We also thank Bill Donnelly for valuable discussions. M.J.S. would like to thank the Center for Theoretical Physics at MIT for kind hospitality during the completion of this work. The work of M.J.S. is supported in part by the US Department of Energy under Grant No. DE-FG03-97ER4014. The work of S.R.B. is supported in part by the National Science Foundation under grant No. PHY-0400231 and by DOE contract DE-AC05-84ER40150, under which the Southeastern Universities Research Association (SURA) operates the Thomas Jefferson National Accelerator Facility.

\section{References}

[1] European Muon Collaboration, J.J. Aubert, et al., Phys. Lett. B 123 (1983) 275.

[2] M. Arneodo, Phys. Rep. 240 (1994) 301.

[3] D.F. Geesaman, K. Saito, A.W. Thomas, Annu. Rev. Nucl. Part. Sci. 45 (1995) 337.

[4] G. Piller, W. Weise, Phys. Rep. 330 (2000) 1, hep-ph/9908230.

[5] P.R. Norton, Rep. Prog. Phys. 66 (2003) 1253.

[6] J.R. Smith, G.A. Miller, Phys. Rev. Lett. 91 (2003) 212301, nucl-th/0308048.

[7] F.M. Steffens, A.W. Thomas, K. Tsushima, Phys. Lett. B 595 (2004) 237, hep-ph/0405096.

[8] J.W. Chen, G. Rupak, M.J. Savage, Nucl. Phys. A 653 (1999) 386, nucl-th/9902056.

[9] D. Arndt, M.J. Savage, Nucl. Phys. A 697 (2002) 429, nucl-th/0105045.

[10] J.W. Chen, X.D. Ji, Phys. Lett. B 523 (2001) 107, hep-ph/0105197.

[11] J.W. Chen, X.D. Ji, Phys. Lett. B 523 (2001) 73, hep-ph/0105296.

[12] J.W. Chen, X.D. Ji, Phys. Rev. Lett. 87 (2001) 152002, hep-ph/0107158; J.W. Chen, X.D. Ji, Phys. Rev. Lett. 88 (2002) 249901, Erratum.

[13] J.W. Chen, X.D. Ji, Phys. Rev. Lett. 88 (2002) 052003, hep-ph/0111048.

[14] J.W. Chen, M.J. Savage, Nucl. Phys. A 707 (2002) 452, nucl-th/0108042.

[15] J.W. Chen, M.J. Savage, Phys. Rev. D 65 (2002) 094001, hep-lat/0111050.

[16] S.R. Beane, M.J. Savage, Nucl. Phys. A 709 (2002) 319, hep-lat/0203003.

[17] A.V. Belitsky, X. Ji, Phys. Lett. B 538 (2002) 289, hep-ph/0203276.

[18] P.A.M. Guichon, L. Mosse, M. Vanderhaeghen, Phys. Rev. D 68 (2003) 034018, hep-ph/0305231.

[19] J.W. Chen, M.J. Savage, Nucl. Phys. A 735 (2004) 441, nucl-th/0308033.

[20] N. Kivel, M.V. Polyakov, S. Stratmann, nucl-th/0407052.

[21] M. Butler, J.W. Chen, Nucl. Phys. A 675 (2000) 575, nucl-th/9905059.

[22] D.B. Kaplan, M.J. Savage, M.B. Wise, Phys. Lett. B 424 (1998) 390, nucl-th/9801034.

[23] D.B. Kaplan, M.J. Savage, M.B. Wise, Nucl. Phys. B 534 (1998) 329, nucl-th/9802075.

[24] D.B. Kaplan, M.J. Savage, M.B. Wise, Phys. Rev. C 59 (1999) 617, nucl-th/9804032.

[25] U. van Kolck, hep-ph/9711222.

[26] D.B. Kaplan, Nucl. Phys. B 494 (1997) 471, nucl-th/9610052.

[27] S.R. Beane, M.J. Savage, Nucl. Phys. A 694 (2001) 511, nucl-th/0011067.

[28] S.I. Ando, C.H. Hyun, nucl-th/0407103.

[29] W. Detmold, M.J. Savage, Nucl. Phys. A 743 (2004) 170, hep-lat/0403005. 
[30] W. Detmold, hep-lat/0410011.

[31] M.M. Sargsian, et al., J. Phys. B 29 (2003) R1, nucl-th/0210025.

[32] A. Kirchner, D. Müller, Eur. Phys. J. C 32 (2003) 347, hep-ph/0302007.

[33] A. Freund, M. Strikman, Eur. Phys. J. C 33 (2004) 53, hep-ph/0309065.

[34] J.W. Chen, M.J. Savage, Phys. Rev. C 60 (1999) 065205, nucl-th/9907042.

[35] G. Rupak, Nucl. Phys. A 678 (2000) 405, nucl-th/9911018.

[36] P.F. Bedaque, H.W. Hammer, U. van Kolck, Nucl. Phys. A 646 (1999) 444, nucl-th/9811046.

[37] P.F. Bedaque, H.W. Hammer, U. van Kolck, Nucl. Phys. A 676 (2000) 357, nucl-th/9906032. 